Document downloaded from:

http://hdl.handle.net/10251/120351

This paper must be cited as:

Salvador-Herranz, G.; Camba, J.; Contero, M.; Naya Sanchis, F. (2018). Accessibility and tangible interaction in distributed workspaces based on multi-touch surfaces. Universal Access in the Information Society. 17(2):247-256. https://doi.org/10.1007/s10209-017-05637

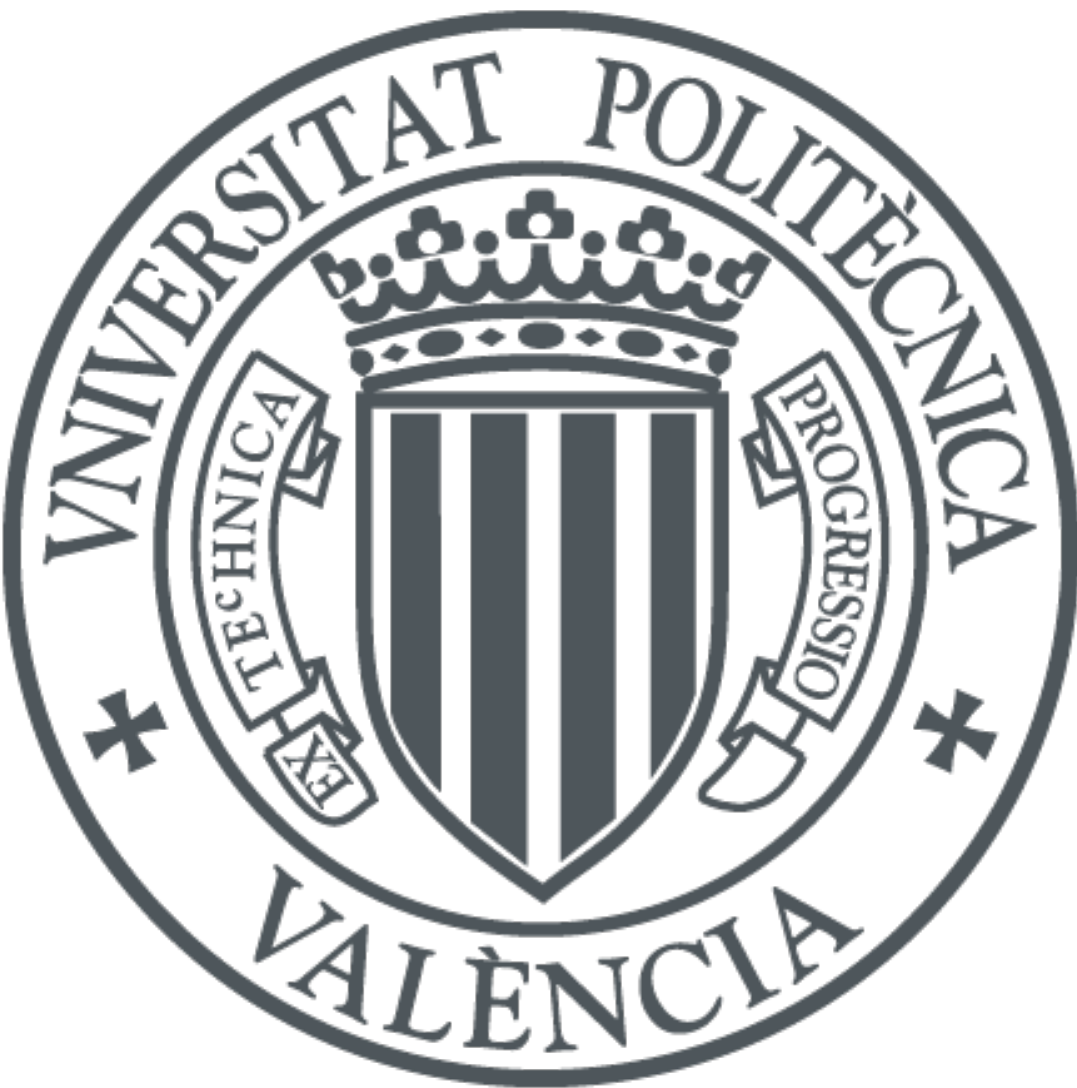

The final publication is available at

http://doi.org/This work was supported by the Spanish Ministry of Economy and Competitiveness and the European Regional Development Fund, through the ANNOTA Project (Ref. TIN2013-46036-C3-1-R).

Copyright Springer Verlag

Additional Information 


\title{
Accessibility and Tangible Interaction in Distributed Workspaces Based on Multi-Touch Surfaces
}

\author{
Gustavo Salvador-Herranz • Jorge D. Camba • Ferran Naya • Manuel \\ Contero
}

Received: date / Accepted: date

\begin{abstract}
Traditional interaction mechanisms in distributed digital spaces often fail to consider the intrinsic properties of action, perception, and communication among workgroups, which may affect access to the common resources used to mutually organize information. By developing suitable spatial geometries and natural interaction mechanisms, distributed spaces can become blended where the physical and virtual boundaries of local and remote spaces merge together to provide the illusion of a single unified space. In this paper, we discuss the importance of blended interaction in distributed spaces and the particular challenges faced when designing accessible technology. We illustrate this discussion through a new tangible interaction mechanism for collaborative spaces based on tabletop system technology implemented with optical frames. Our tangible elements facilitate the exchange of digital information in distributed collaborative settings by providing a physical manifestation of common digital operations. The tangibles are designed as passive elements that do not require the use of any additional hardware or external power while maintaining a high degree of accuracy.
\end{abstract}

G. Salvador-Herranz

ESET, Universidad CEU Cardenal Herrera

San Bartolomé, 55

46115 Alfara del Patriarca, Valencia, Spain

E-mail: gsalva@uchceu.es

J. D. Camba

Gerald D. Hines College of Architecture and Design University of Houston.

Houston, TX

E-mail: jdorribo@uh.edu

F. Naya · M.Contero

Instituto de Investigación en Bioingeniería (I3B). Universitat Politécnica de Valéncia.

Valencia, Spain.

E-mail: fernasan,mcontero@upv.es
Keywords Tangible interaction · Interactive surfaces . Blended spaces · Tabletops

\section{Introduction}

Blended interaction is described as the type of humancomputer interaction where the characteristics and properties of physical and virtual artifacts and practices (e.g. being graspable, squeezable, etc.) are preserved while integrating computing power in a significant and natural manner [25]. In this context, tangible interfaces represent an essential step for developing natural interactions, as they provide mechanisms to manipulate digital information via physical forms based on metaphors for tools and objects we use in our daily lives.

When used in combination with multi-touch interactive surfaces, tangible user interfaces facilitate hybrid or blended spaces, where the manipulation of digital information can be performed by direct touch and finger gestures on the surface or through interaction with the tangible element. Although there has been much discussion regarding the benefits of tangible user interfaces over direct manipulation using multi-touch gestures $[12,29]$, hybrid spaces have been shown effective in computer-supported collaborative work and learning environments because of their ability to facilitate faceto-face communication and promote discussion $[9,17$, $22,23,50]$. Research shows that hybrid spaces can enhance the pre-existing motor, spatial, social, and cognitive skills of groups of users by hiding the complexity of the technology and providing a shareable interface that simulates a traditional work table to allow several people to interact on a common task using their own device $[2,21,31]$. Interfaces based on tangible elements can contribute to remove the barrier to access and ma- 
nipulate digital information by groups that are traditionally not familiar with technology, such as children or elderly users $[15,30,36]$.

The addition of tangible elements to collaborative workspaces becomes particularly relevant (and challenging) in distributed settings where users may manipulate and exchange information with other users and groups that may be geographically dispersed and use different devices and technology. In this paper, we present a tangible interaction mechanism for these hybrid spaces based on multi-touch surfaces implemented with optical frames and discuss the software infrastructure and protocols to facilitate accessibility through various platforms, metaphors, and devices.

The incorporation of fiducial marks on the tangibles is also discussed as a method to expand the orchestration possibilities of distributed workspaces on projection-based tabletop systems.

\section{Related work}

In the past, a growing body of research has explored the concepts of tangible user interfaces (TUIs) [2, 9, 17, 21$23,27,31,50]$ as well as multi-touch interactive surfaces $[8,40,42]$. In the field of TUIs, researchers have tried to describe, classify, and categorize TUIs and explain why these interfaces might offer benefits over more traditional graphical user interfaces (GUIs) [20]. For many users, TUIs are more natural and engaging, as they draw upon the impulse to be active with ones hands [49], and provide a familiar method to interact with a computer that is similar to the way users interact with the non-digital world [24].

The origins of TUIs are closely tied to developments in the areas of augmented reality and ubiquitous computing [41]. The connection is rooted on the idea of retaining the richness of physical interaction and enabling fluid transitions between the digital and the real, as opposed to distancing users from their natural environment as traditional graphical user interfaces and virtual reality environments do $[41,48]$. Early influential examples of TUI include the graspable interfaces developed by Fitzmaurice et al., [13] and Hiroshi Ishiis tangible bits [23]. Since then, much research has focused on the development of domain-specific systems that explore the applications and technical possibilities of TUIs, such as collaborative learning $[43,44,50]$, video logging [6], embodied user interfaces [10,11], music and artistic expression $[27,32,33,47]$, and information visualization $[7,18]$.

In many cases, tangible interfaces use a tabletop surface as a basis for interaction, embedding the tracking
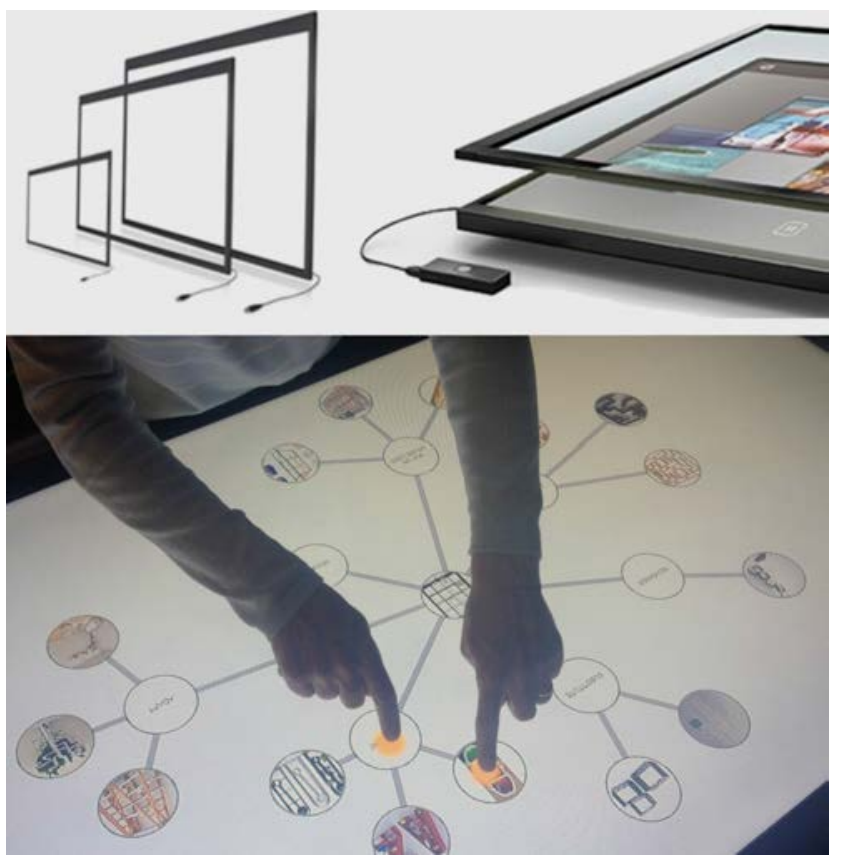

Fig. 1 Multi-touch optical frames based on infrared technology

mechanism in the surface [41]. When TUIs are combined with multi-touch surface technologies, the notion of tangible tabletop interaction emerges. Researchers in this field have investigated the used of tangibles on multi-touch tables [29,38], the differences between touchbased vs. and tangible-based interaction in blended environments [45], and the applications of blended interaction (i.e., tangible + multi-touch input) [27].

Multi-touch surfaces are typically found in tabletop format (although a variety of implementations and form factors have been used [40]). Infrared optical frame technology provides an interesting option for implementation, as any existing flat screen (projected/rear projected or liquid crystal display (LCD)) can be converted into a multi-touch tabletop device type [3] by simply attaching a frame to the screen or projection surface (Fig. $1)$.

In addition, the cost of infrared optical frame technology has drastically been reduced in recent years and the frames are now available in formats and sizes that can fit standard monitors and flat TV screens (including extremely large ones). The high refresh rate $(\geq 200 \mathrm{~Hz})$ as well as the accuracy in detecting touches combined with the ability to process reasonably large numbers of simultaneous actions, make this technology a competitive alternative to implement high-performance tabletop systems, particularly when used in conjunction with large high-resolution displays. Infrared touch-screens use an array of XY infrared LEDs and pairs of photodetectors located at the edges. These photodetectors are re- 


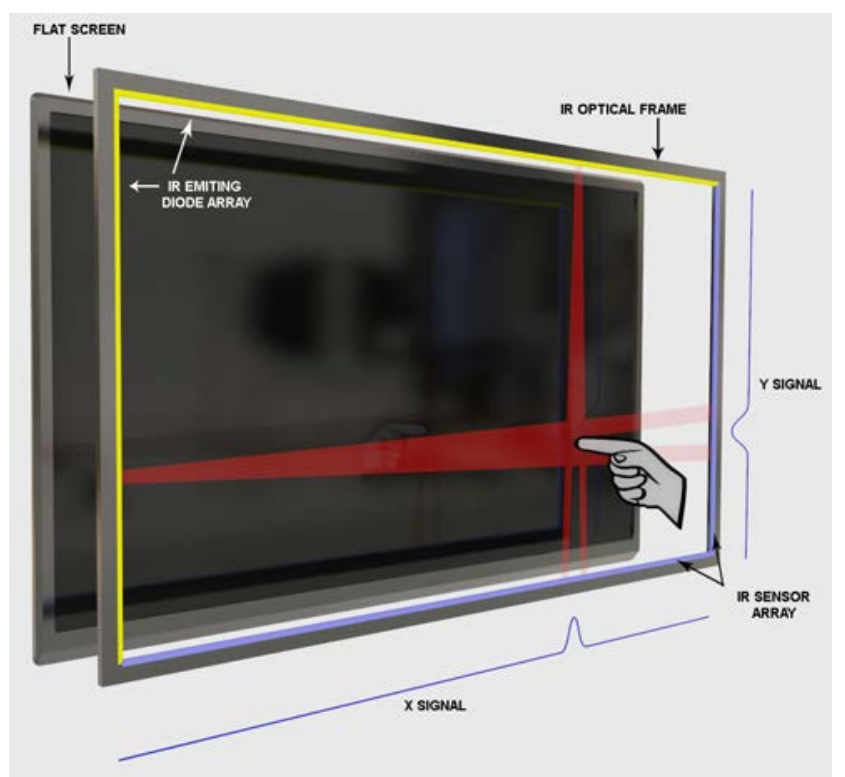

Fig. 2 Infrared technology in optical frame [3]

sponsible for detecting disruptions in the light pattern created by the matrix, as shown in Fig. 2. Disruptions can be triggered by finger input or by any object that interrupts the infrared light beam.

From a technical standpoint, each physical manipulator in a tangible tabletop interaction environment needs to have a unique identifier (ID), so the tangible can be recognized by the system and its position and orientation on the surface can be tracked. In projectionbased tabletops, for example, tracking is typically accomplished by a camera placed underneath the surface that can recognize predefined marks printed at the bottom of the tangibles. These marks are visible from the camera position and through the translucent surface $[27,34]$.

Other implementations have used radiofrequency technology. In this case, each tangible is equipped with an RFID card where an LC circuit resonates at a particular frequency. The position of the manipulator on the surface is determined by triangulating the resonance values generated by the RFID module on different antennas disposed around the tabletop device. However, only the XY position of the tangible can be determined, as calculating its orientation requires installing additional RFID cards on the tangible, which can significantly increase the cost and complexity of the tabletop solution [19].

In this paper, we expand our previous work on tangible interaction in multi-touch surface environments [38] by describing the technical infrastructure and tangible interaction mechanisms of a system designed to support collaborative work in distributed settings and provide accessibility throughout devices. Our approach uses custom-built tangible elements to interrupt the infrared light pattern in the optical frame by simulating finger gestures that can be used to trigger specific actions.

\section{Design of passive tangible manipulators}

The tangible manipulators in our system are cylindrical objects with a series of small pins attached to the bottom face of the form. When the manipulator is placed on the interactive surface, the pins interrupt the light beam generated by the optical frame (Fig. 4). The number and position of these pins are used to identify the manipulator and determine its position and orientation on the surface. The manipulators are designed to be cost-effective and easy to manufacture (e.g., they can be easily $3 \mathrm{D}$ printed from a computer model).

A minimum of three pins are required to create a valid manipulator. The pins define the three vertices of a triangle, which make the tangible manipulator stable on the interactive surface (see identifier '0' in Fig. 3). Furthermore, the pins need to be arranged in a manner that defines an isosceles triangle. The two longer sides of this isosceles triangle are used to determine the position of the manipulator (relative to its center point) as well as its orientation. Additional pins located along the shorter side of the triangle are used to encode a unique identifier (ID) in binary form. In the example shown in Figure 4, three pins (located between the two pins that define the shortest side of the triangle) are used to encode eight different IDs: 0 (000 in binary), 1 (001 in binary), 2 (010 in binary), 3 (011 in binary), 4 (100 in binary), 5 (101 in binary), 6 (110 in binary) and 7 (111 in binary).

The proposed method allows the encoding of $2^{\mathrm{n}}$ identifiers, where ' $n$ ' is the maximum number of pins that can be placed between the two pins that define the shortest side of the isosceles triangle. The maximum number of pins is limited by the resolution of the optical frame: the higher the resolution, the higher number of pins (the more unique IDs that can be encoded). However, more pins can be added by simply creating more space on the physical manipulator, i.e., by increasing the radius (and therefore the overall size) of the cylindrical form.

\subsection{Position Tracking}

To determine the position of the tangible manipulator on the interactive surface, information clustering techniques are applied. The set of XY points corresponding 

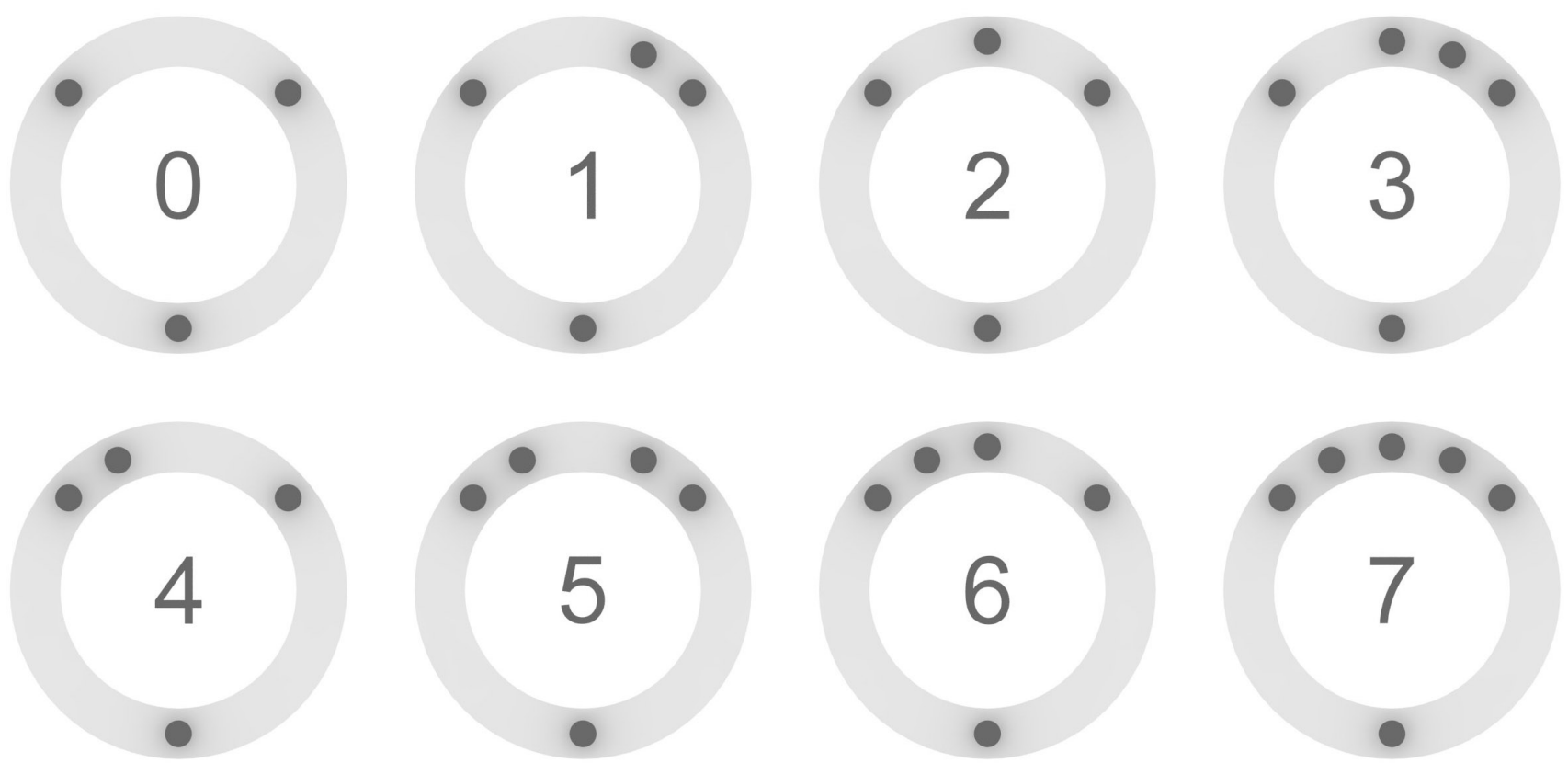

Fig. 3 Binary encoding of IDs between the two pins that define the shorter side of the triangle

to the positions of the pins on the tangible are interpreted as simultaneous touch events on the surface and processed as point clouds, which can be automatically grouped together.

Functionality to detect touch events on an interactive surface is typically available via the Application Programming Interface (API) and gesture tracking libraries of the tabletop system. Most commercial optical frames make their APIs available, where basic functions to program the hardware are provided to facilitate software development. In our implementation, a PQ Labs optical frame (sampling rate of 200fps) was selected. Once the frame is properly calibrated, the number of simultaneous touches on the system and their XY positions in screen units can be retrieved automatically by a set of functions that relate the optical frame to the screen system, typically through a homography matrix.

The general structure of a typical software application that uses the API of an optical frame is described in Fig. 5. In particular, the callback functions of received data are automatically triggered whenever a touch or gesture is detected. In the case of tangible manipulators, only the detection of new touches is considered.

To determine the clusters, the Quality Threshold (QT) algorithm is applied [26]. Although the QT algorithm is computationally intensive, particularly for realtime applications, the number of items to be processed is relatively small (the maximum number of simultaneous touches supported by current optical frames is typically 30-50). Unlike other clustering algorithms such as the K-Means [16], the QT algorithm requires no ini- tial presetting of the number of clusters to form, but the maximum distance between two points to determine whether they belong to the same cluster. From this input, the QT algorithm automatically detects the number of clusters and classifies the points. In the case of the point clouds produced by the tangible manipulators, the maximum distance between points is the diameter of the circle that defines the manipulator (plus a certain error threshold value).

As an example, the scenario shown in Fig. 6a illustrates a situation where three tangible manipulators (corresponding to encodings 2, 3, and 5 in the set from Fig. 3) and three finger touches are simultaneously detected on a surface. The corresponding clusters that were identified by QT algorithm are shown in Fig. 6b. Since each manipulator has at least three pins, any cluster with less than three points is immediately discarded for further analysis. Therefore, in the situation shown in Figure 6b, only the three clusters highlighted in blue will be identified as tangibles. Nevertheless, the two clusters that were detected by the algorithm but not identified as being produced by a tangible manipulator are still processed as regular touch events. This combination of tangibles and regular finger gestures within the same environment facilitates the implementation of blended interaction mechanisms.

\subsection{Orientation Tracking}

Once all potential clusters have been located, the convex polygon that contains the corresponding points is 

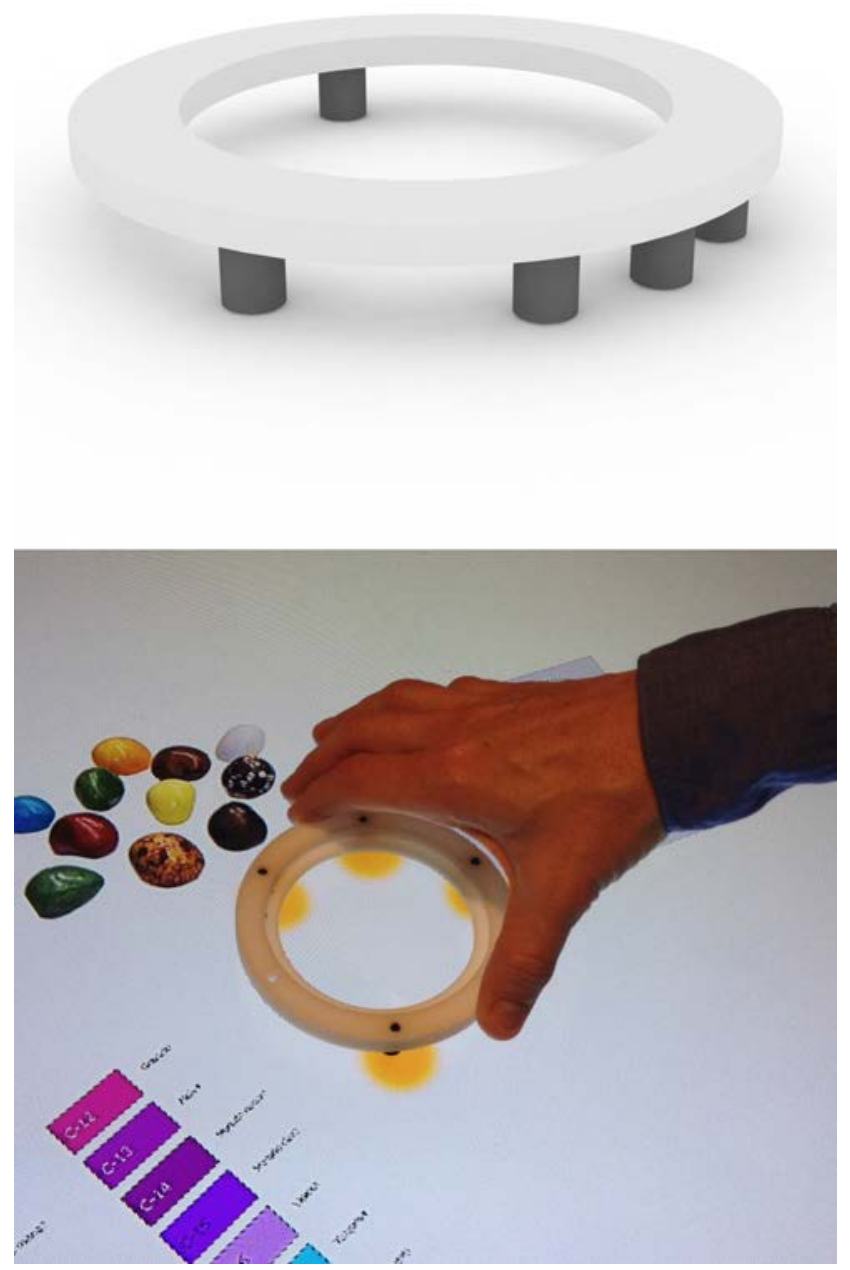

Fig. 4 3D computer model (top) and physical tangible used on a tabletop (bottom)

calculated [14], as shown in Fig. 7a. Next, vectors of the two longer sides of the polygon are determined: (v1v2) and (v1v4) in Fig. 7b. Finally, the direction vector of the manipulator is calculated by adding these two vectors. The center $\mathrm{C}$ of the manipulator can be obtained by converting the direction vector in its corresponding unit vector and multiplying it by the radius of tangible (Fig. 7c).

\subsection{ID Tracking}

To determine the unique ID of the tangible element, shape matching techniques are employed [46]. The algorithm described by Arkin et al. [1] is particularly useful, as it is not affected by the rotation of the polygon or variations of scale or reference point. The algorithm

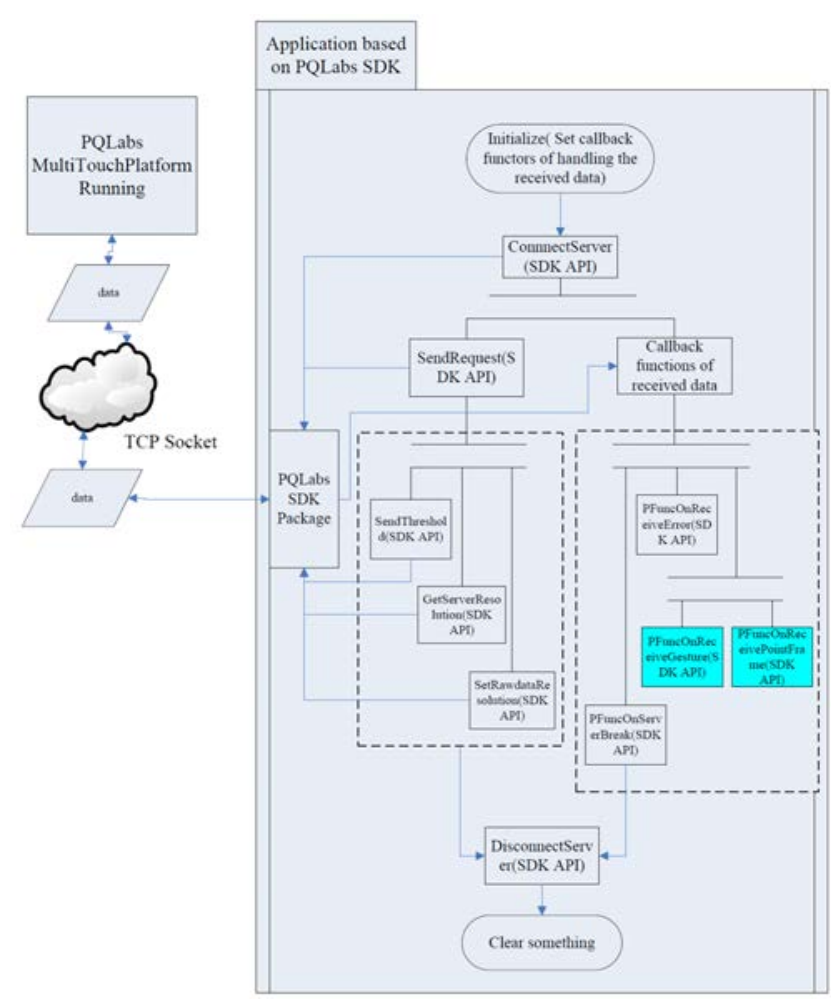

Fig. 5 General structure of software application based on the API of PQ Labs Optical Frame [35].

allows real-time identification of the polygon associated to a tangible manipulator at a reasonable computational cost. Depending on the number of pins, the system will store the collection of possible polygons in a database and assign an identifier to each one according to the binary system described earlier. Examples of the possible polygons available for a 2-pin manipulator (four possible combinations) are shown in Fig. 8.

By comparing the form retrieved from the cluster with the polygons stored in the database, the system can assign an identifier to the corresponding tangible manipulator. If a matching shape is not found, no manipulator is assigned to that particular set of points, which is then handled as conventional touches or gestures.

\subsection{Information Processing}

Once a tangible manipulator is fully identified, the system encapsulates its associated information ID, position (center point), and orientation (direction vector) in TUIO frames, which are then broadcasted via UDP/IP (or TCP/IP) through port 3333 .

This encapsulation is performed by an intermediate software layer or middleware, whose basic structure is illustrated in Fig. 9. From a software developers stand- 


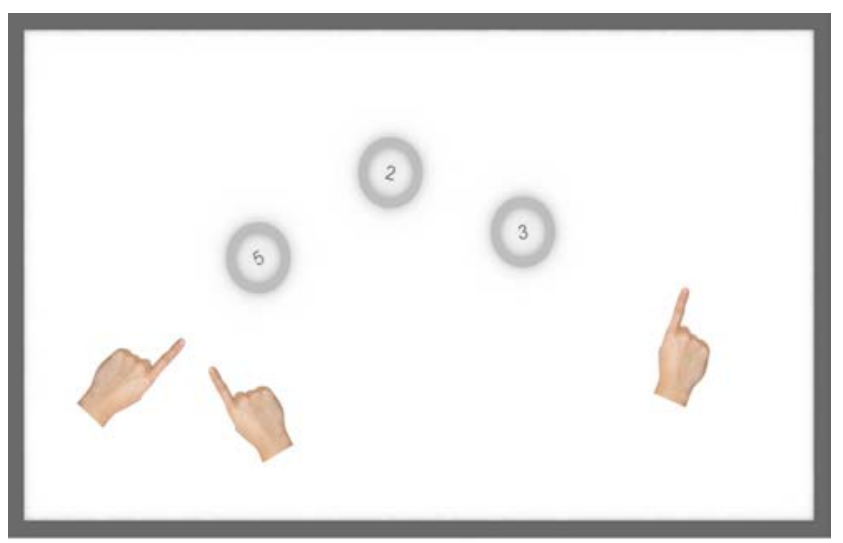

(a)

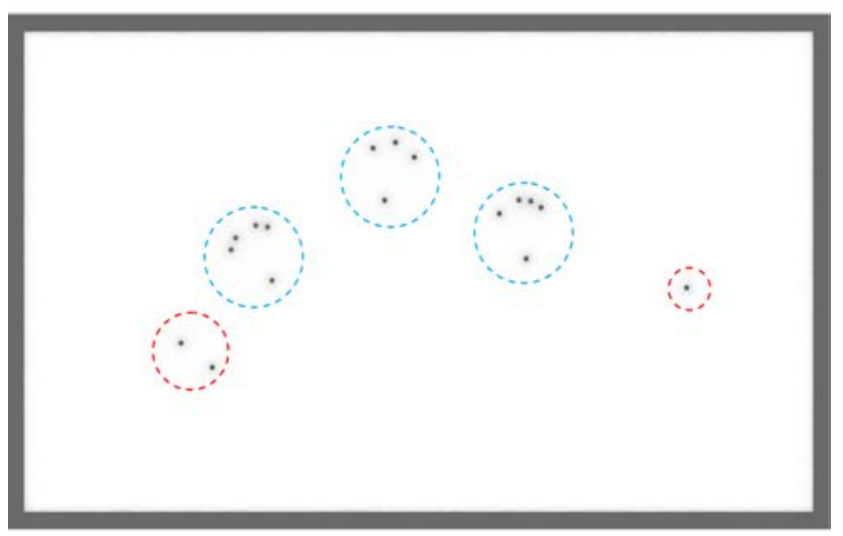

(b)

Fig. 6 Three tangible manipulators and three finger touches simultaneously detected on a surface (a), and corresponding clusters identified by the QT algorithm (b).

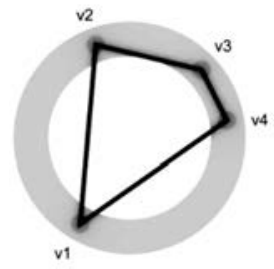

(a)

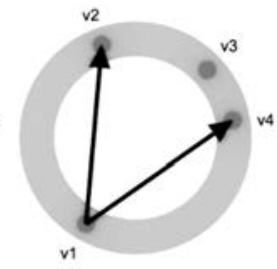

(b)

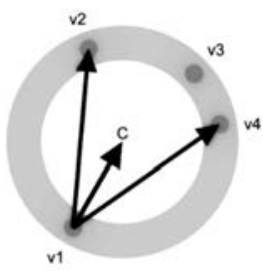

(c)
Fig. 7 Determining the position and orientation of a tangible manipulator: convex polygon (a), vectors of two longer sides (b), and direction vector (c).

point, all TUIO frames are handled equally [28], regardless of whether they encapsulate simple finger touches or information associated with tangible physical manipulators.

The structure of a frame TUIO is described in Table 1. The following parameters are encoded: session ID, class ID, position and angle (only for manipulators). Class ID (integer) encodes the TUIO object type. In

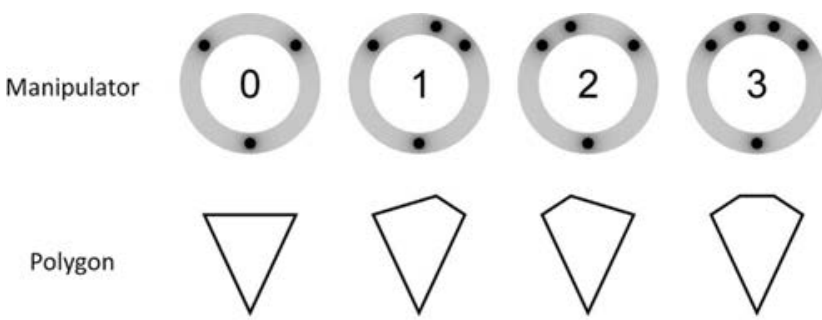

Fig. 8 Polygons associated to four manipulators and identified by their pins.

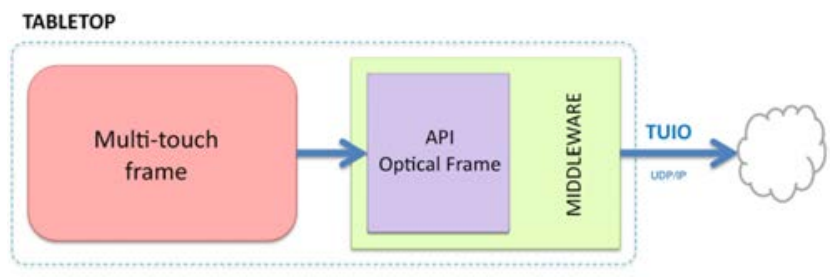

Fig. 9 Middleware structure.

Table 1 Parameters of a TUIO packet

\begin{tabular}{ll} 
Parameter & Description \\
\hline $\mathrm{s}$ & Session ID (temporary object ID) \\
$\mathrm{i}$ & Class ID (e.g. marker ID) \\
$\mathrm{x}, \mathrm{y}, \mathrm{z}$ & Position \\
$\mathrm{a}, \mathrm{b}, \mathrm{c}$ & Angle \\
$\mathrm{w}, \mathrm{h}, \mathrm{d}$ & Dimension \\
$\mathrm{f}, \mathrm{v}$ & Area, Volume \\
$\mathrm{X}, \mathrm{Y}, \mathrm{Z}$ & Velocity vector (motion speed and direction) \\
$\mathrm{A}, \mathrm{B}, \mathrm{C}$ & Rotation velocity vector (rotation speed and direction) \\
$\mathrm{m}$ & Motion acceleration \\
$\mathrm{r}$ & Rotation acceleration \\
$\mathrm{p}$ & Free parameter \\
\hline
\end{tabular}

our implementation, a 0 represents a single touch on the tabletop; the range 1-10, represents the tangible manipulators (a maximum of ten); and the range 11-20 represent fiducials marks (discussed in the next section). For a given class, Session ID stores a unique identifier for each instance of that TUIO type on the tabletop. Position consists of three floating point numbers that track the $\mathrm{x}, \mathrm{y}, \mathrm{z}$ coordinates of the object on the board. In our implementation, the $\mathrm{z}$ coordinate is always 0 unless fiducial marks are used. The orientation of a tangible manipulator or a fiducial mark is tracked by the Angle parameter. For tangibles, only the rotation around the Z-axis (normal to the tabletop) is encoded.

\subsection{Integration of Fiducial Marks}

To facilitate accessibility and information exchange in collaborative workspaces and distributed learning environments, where projection based systems and optical frame interactive surfaces may coexist, a hybrid model of tangible interaction is proposed. 


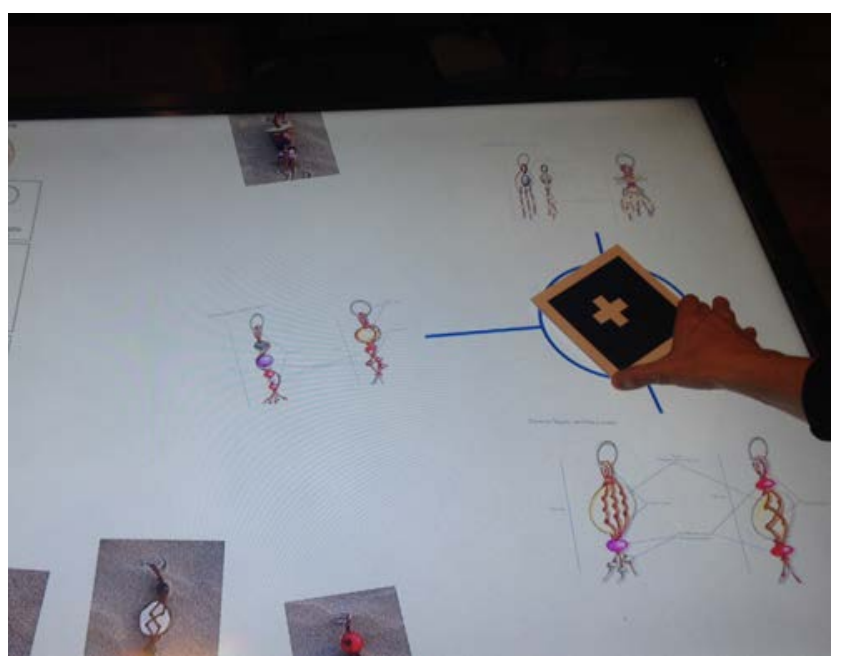

Fig. 10 Fiducial mark as tangible element.

In this model, the tangible elements of both systems are combined into a single manipulator that can be used interchangeably. This integration simplifies workflows between various working groups that may use different technologies and promotes accessibility.

Fiducial marks are tangible elements that can be used in projection based tabletops (Fig. 10). These marks are inexpensive and easy to manufacture (they can be printed on paper and attached to a cardboard sheet or a thin wooden piece). Visibility problems may arise, however, when the mark is fully or partially covered by the users hand, although usability tests have revealed that users intuitively adjust to the interaction by slightly modifying the way they hold and manipulate the mark.

To implement interaction with fiducial marks, a camera must be placed above the tabletop system, pointing to the surface. The camera optics should allow for adjustments of the focal length so the captured image can pick up the entire surface based on the height at which the camera is installed. A high-speed camera is preferred, although certain medium/high-end web cameras can also meet the demands.

A calibration process is required prior to using fiducial marks to correlate the positions of the marks on the tabletop with their positions on the reference system of the image captured by the camera. This calibration process is performed by projecting a checkerboard pattern on the surface, which is used by the OpenCV computer vision library [4] to correct camera distortion parameters (both intrinsic and extrinsic) and adjust the perspective. Once the system is calibrated, custom software is used for the recognition of fiducial marks [5].

Hybrid manipulators can be created by attaching the printed fiducial mark onto the top surface of the tangible element used for optical frames (see Fig. 11).

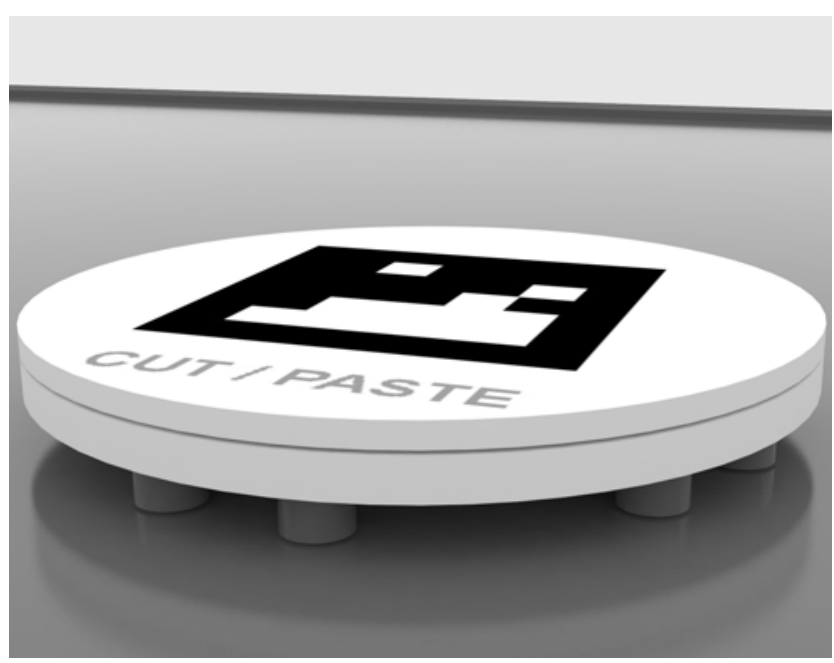

Fig. 11 Hybrid tangible manipulator.

The system encodes TUIO frames which are then broadcasted via UDP through port 3333, as described earlier. Fiducial marks are classified with a Class ID between 11 and 20 (which allows the use of ten different fiducial marks).

\section{Tangible interaction in collaborative workspaces}

The distributed architecture of collaborative workspaces allows teamwork in a co-localized manner. This means that multiple users (who may be geographically dispersed) can communicate and work together in real time using a common virtual space. In this context, the incorporation of tangible manipulators can simplify basic interface and information management tasks by making them more natural and intuitive and actively generating a mixed reality environment. The operations and actions associated with tangibles do not replace existing interaction mechanisms, but complement the information management tools based on gestures that are already implemented by multi-touch surfaces. Some examples of the use of tangible elements in collaborative scenarios are discussed in our previous work [37-39].

\subsection{Work Plane Manipulation}

Zooming and panning operations can be performed by a single tangible manipulator, as it is not necessary to manually lock and unlock the workspace (to prevent involuntary actions) or to select tools from a menu. By placing the tangible associated with these actions on the multi-touch surface, the workspace becomes automatically unlocked and ready to be manipulated. Removal 
from the surface causes the virtual workspace to re-lock. Furthermore, the tangible can be placed anywhere on the surface, even over active information items. Once on the surface, the tangible can be moved in any direction. These actions are transferred to the camera associated to the device on the working plane which controls the framing. Similarly, rotating the tangible manipulator clockwise causes an increase in the focal length of the virtual camera, which translates into a magnifying zoom effect. Anti-clockwise rotations cause the image to zoom out.

\subsection{Digital Information in Local Mode}

Operations related to information storage can be associated to tangible manipulators in local mode, where the tangibles can be linked to digital information. When a tangible is placed on the multi-touch surface, an indicator (displayed as a circle around the object, as shown in Figure 10) will appear on the surface, where it becomes an active element that can be used to connect information items by drag-and-drop actions. The digital information that is linked to a tangible will disappear from the screen when the tangible is removed from the surface. When the tangible is placed back on the tabletop, the digital information that is linked to it will reappear next to the manipulator and at the same representation scale. New information items can be added or retrieved at any time. In addition, if the parent node in a hierarchical relationship is linked to a tangible manipulator, the entire structure (the parent node plus all the child nodes) will be connected to the physical element, as hierarchical structures are handled as single information items by our system. Similarly, the operation to remove information from the system is also available in local mode. Digital information items can be linked to the tangible associated with the delete operation by drag-and-drop actions. A dotted red line is displayed to warn users about the operation.

\subsection{Digital Information in Distributed Mode}

In a distributed collaborative work environment with multiple interactive surfaces (some of which may be geographically dispersed), it is essential to provide a simple and natural interaction mechanism to exchange information and create a blended space, where the physical and virtual boundaries of local and remote spaces merge together to provide the illusion of a single unified space. For example, copy/cut and paste operations (which are typically available via finger gestures) can be easily implemented through tangible manipulators

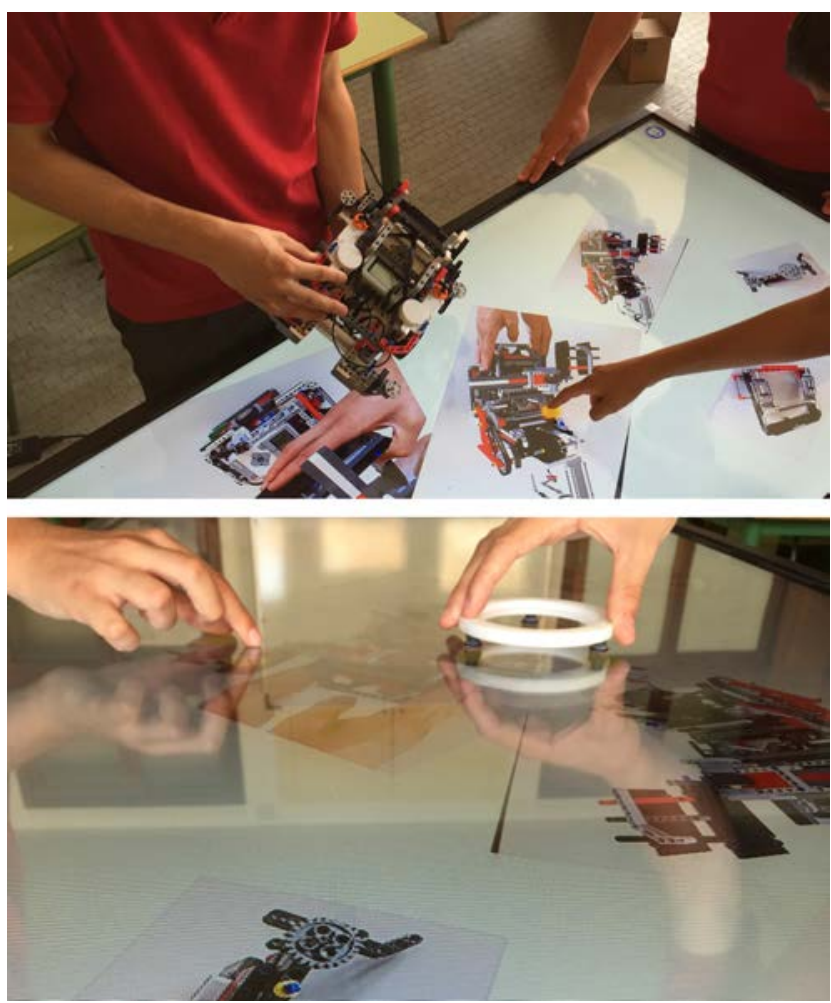

Fig. 12 Students working collaboratively with passive tangible elements in a distributed scenario implemented by optical frame tabletops

(Figure 11). By placing the associated manipulator over a digital item displayed on the surface of the tabletop, users can use a physical action to copy or cut information. This information is temporarily stored in a clipboard on the application server. When the manipulator is placed on the same or a different tabletop, the information is transferred from the clipboard to that surface.

In our system, all operations related to tangibles are global, i.e., they can be used between distributed workgroups. For example, if two computers (users) connected to an application server use the same tangible manipulator (same ID) for the copy and paste operation, one user can begin to copy information from the first surface and the other can paste it onto the second surface by using her own tangible. This style of work facilitates accessibility to information and promotes the understanding of the physical manipulation of information.

As an example of the system in action, a pilot test is shown in Fig. 12 where a group of students organized in two teams worked collaboratively to design a mobile robot. The distributed digital work space was implemented by optical frame tabletop devices using the technology developed by our research group and described in our previous work [37]. The system allows the creation of shared two-dimensional workspaces 
where tabletops represent working windows. Users can interact freely on the digital plane of their respective working areas, which can also be shared among different groups. In a first session, the two groups of users were allowed to exchange information using the basic operations offered by the system (which are based on Natural Interfaces) without the use of tangibles. In a second session, students were allowed to use tangible elements to exchange information between the two tables. Interviews were conducted after the experience to discuss usability aspects of the interaction. Participants agreed that the exchange of information between tabletops was less confusing when tangible elements were used. The action was also more natural due to its similarity with the real action of transferring physical information between work tables.

\section{Conclusions and Future Work}

The availability and affordability of large-format displays and TV screens combined with the development of multi-touch interactive technology based on high resolution optical frames provides a viable option for the implementation of tabletop devices with large and accurate interactive surfaces. In this paper, we have presented a novel interaction mechanism based on the design and implementation of a new passive type of tangible manipulator that is capable of operating on interactive surfaces built with optical frames.

Tangible elements facilitate the integration of interactive surfaces based on tabletop devices into collaborative workspaces, which in turn generate distributed mixed reality environments rooted on the concept of ubiquitous computing. The incorporation of tangible elements facilitates the sharing and exchange of digital information among devices by providing a physical manifestation of common digital operations. Our proposed solution is designed to be used in tabletop systems based on optical frames. It is a passive solution that capitalizes on the existing hardware and does not require any additional components. The implementation is simple and inexpensive, as the tangibles can be easily $3 \mathrm{D}$ printed from geometric models.

In order to create learning environments and workspaces that are closer to the concept of mixed reality, a blended environment is suggested, where both projection-based devices and optical frame tabletops are used simultaneously. For these scenarios, a hybrid tangible manipulator has been proposed that is compatible with both systems. The new manipulator allows the manipulation and exchange of information between the two and facilitates accessibility across devices. Additionally, an intermediate software layer (middleware) was developed to integrate the proposed tangibles within the distributed collaborative work model by abstracting and encapsulating interaction events into frames of the TUIO communications protocol.

As future work, we are interested in developing and exploring additional types of tangible manipulators, so more complex actions can be implemented. For example, the addition of functional buttons or switches and the ability to remotely control a tangible under certain conditions may significantly expand the interaction possibilities of our system. Finally, comprehensive usability studies in the context of collaborative work and learning are also in progress. In this regard, the system must be evaluated more rigorously so that statistical information can be analyzed with the appropriate degree of significance. This evaluation requires comprehensive experiences over time and the participation of a larger and more diverse number of users. Some experiences focused on the evaluation of three parameters: efficiency, usability and motivation, are currently in progress or under developement. For these tasks, the use of quasiexperimental designs, pre-post usability tests, and prepost Intrinsic Motivation Inventory (IMI) tests, as well as interviews and post analysis of recorded sessions are being used. Results will be reported in future studies.

Acknowledgements This work was supported by the Spanish Ministry of Economy and Competitiveness and the European Regional Development Fund, through the ANNOTA project (Ref. TIN2013-46036-C3-1-R).

\section{References}

1. Arkin, E.M., Chew, L.P., Huttenlocher, D.P., Kedem, K., SB, J.: An efficiently computable metric for comparing polygonal shapes. IEEE Transactions on Acoustics Speech and Signal Processing 13(3), 0 (1991)

2. Benyon, D.: Presence in blended spaces. Interacting with Computers 24(4), 219-226 (2012)

3. Bhalla, M.R., Bhalla, A.V.: Comparative study of various touchscreen technologies. International Journal of Computer Applications 6(8), 12-18 (2010)

4. Bradski, G., Kaehler, A.: Learning OpenCV: Computer vision with the OpenCV library. "O'Reilly Media, Inc." (2008)

5. Candela, E.S., Pérez, M.O., Romero, C.M., López, D.C.P., Herranz, G.S., Contero, M., Raya, M.A.: Humantop: a multi-object tracking tabletop. Multimedia tools and applications 70(3), 1837-1868 (2014)

6. Cohen, J., Withgott, M., Piernot, P.: Logjam: a tangible multi-person interface for video logging. In: Proceedings of the SIGCHI conference on Human Factors in Computing Systems, pp. 128-135. ACM (1999)

7. Couture, N., Rivière, G., Reuter, P.: Geotui: a tangible user interface for geoscience. In: Proceedings of the 2nd international conference on Tangible and embedded interaction, pp. 89-96. ACM (2008) 
8. Dietz, P., Leigh, D.: Diamondtouch: a multi-user touch technology. In: Proceedings of the 14th annual ACM symposium on User interface software and technology, pp. 219-226. ACM (2001)

9. Falcão, T.P., Price, S.: What have you done! the role of'interference'in tangible environments for supporting collaborative learning. In: Proceedings of the 9th international conference on Computer supported collaborative learning-Volume 1, pp. 325-334. International Society of the Learning Sciences (2009)

10. Fallman, D.: Wear, point and tilt. In: Proceedings of the Conference on Designing Interactive Systems: Processes, Practices, Methods, and Techniques. ACM Press, pp. 293-302 (2002)

11. Fishkin, K.P., Gujar, A., Harrison, B.L., Moran, T.P., Want, R.: Embodied user interfaces for really direct manipulation. Communications of the ACM 43(9), 74-80 (2000)

12. Fitzmaurice, G.W., Buxton, W.: An empirical evaluation of graspable user interfaces: towards specialized, spacemultiplexed input. In: Proceedings of the ACM SIGCHI Conference on Human factors in computing systems, pp. 43-50. ACM (1997)

13. Fitzmaurice, G.W., Ishii, H., Buxton, W.A.: Bricks: laying the foundations for graspable user interfaces. In: Proceedings of the SIGCHI conference on Human factors in computing systems, pp. 442-449. ACM Press/AddisonWesley Publishing Co. (1995)

14. Graham, R.L., Yao, F.F.: Finding the convex hull of a simple polygon. Journal of Algorithms 4(4), 324-331 (1983)

15. de la Guía, E., Lozano, M.D., Penichet, V.R.: Cognitive rehabilitation based on collaborative and tangible computer games. In: Pervasive Computing Technologies for Healthcare (PervasiveHealth), 2013 7th International Conference on, pp. 389-392. IEEE (2013)

16. Hartigan, J.A., Wong, M.A.: Algorithm as 136: A kmeans clustering algorithm. Journal of the Royal Statistical Society. Series C (Applied Statistics) 28(1), 100-108 (1979)

17. Higgins, S.E., Mercier, E., Burd, E., Hatch, A.: Multitouch tables and the relationship with collaborative classroom pedagogies: A synthetic review. International Journal of Computer-Supported Collaborative Learning 6(4), 515-538 (2011)

18. Hinckley, K., Pausch, R., Goble, J.C., Kassell, N.F.: Passive real-world interface props for neurosurgical visualization. In: Proceedings of the SIGCHI conference on Human factors in computing systems, pp. 452-458. ACM (1994)

19. Hinske, S.: Determining the position and orientation of multi-tagged objects using rfid technology. In: Pervasive Computing and Communications Workshops, 2007. PerCom Workshops' 07. Fifth Annual IEEE International Conference on, pp. 377-381. IEEE (2007)

20. Hornecker, E.: A design theme for tangible interaction: embodied facilitation. In: ECSCW 2005, pp. 23-43. Springer (2005)

21. Hoshi, K., Öhberg, F., Nyberg, A.: Designing blended reality space: conceptual foundations and applications. In: Proceedings of the 25th BCS Conference on HumanComputer Interaction, pp. 217-226. British Computer Society (2011)

22. Ishii, H.: Tangible user interfaces. CRC Press (2007)

23. Ishii, H., Ullmer, B.: Tangible bits: towards seamless interfaces between people, bits and atoms. In: Proceedings of the ACM SIGCHI Conference on Human factors in computing systems, pp. 234-241. ACM (1997)

24. Jacob, R.J., Girouard, A., Hirshfield, L.M., Horn, M.S. Shaer, O., Solovey, E.T., Zigelbaum, J.: Reality-based interaction: a framework for post-wimp interfaces. In: Proceedings of the SIGCHI conference on Human factors in computing systems, pp. 201-210. ACM (2008)

25. Jetter, H.C., Dachselt, R., Reiterer, H., Quigley, A., Benyon, D., Haller, M.: Blended interaction: envisioning future collaborative interactive spaces. ACM (2013)

26. Jin, X., Han, J.: Quality threshold clustering. In: Encyclopedia of Machine Learning, pp. 820-820. Springer (2011)

27. Jordà, S., Geiger, G., Alonso, M., Kaltenbrunner, M.: The reactable: exploring the synergy between live music performance and tabletop tangible interfaces. In: Proceedings of the 1st international conference on Tangible and embedded interaction, pp. 139-146. ACM (2007)

28. Kaltenbrunner, M., Bovermann, T., Bencina, R., Costanza, E.: Tuio: A protocol for table-top tangible user interfaces. In: Proc. of the The 6th Intl Workshop on Gesture in Human-Computer Interaction and Simulation, pp. $1-5(2005)$

29. Kirk, D., Sellen, A., Taylor, S., Villar, N., Izadi, S.: Putting the physical into the digital: issues in designing hybrid interactive surfaces. In: Proceedings of the 23rd British HCI Group Annual Conference on People and Computers: Celebrating People and Technology, pp. 35-44. British Computer Society (2009)

30. Marques, T., Nunes, F., Silva, P., Rodrigues, R.: Tangible interaction on tabletops for elderly people. In: International Conference on Entertainment Computing, pp. 440-443. Springer (2011)

31. Müller, D.: Mixed reality systems. iJOE 5(S2), 10-11 (2009)

32. Newton-Dunn, H., Nakano, H., Gibson, J.: Block jam: a tangible interface for interactive music. In: Proceedings of the 2003 conference on New interfaces for musical expression, pp. 170-177. National University of Singapore (2003)

33. Patten, J., Recht, B., Ishii, H.: Audiopad: a tag-based interface for musical performance. In: Proceedings of the 2002 conference on New interfaces for musical expression, pp. 1-6. National University of Singapore (2002)

34. Patten, J., Recht, B., Ishii, H.: Interaction techniques for musical performance with tabletop tangible interfaces. In: Proceedings of the 2006 ACM SIGCHI international conference on Advances in computer entertainment technology, p. 27. ACM (2006)

35. PQLabs: Inc.http://multitouch.com/ (retrieved on 10/16/2016).

36. Ryokai, K., Marti, S., Ishii, H.: I/o brush: Drawing with everyday objects as ink. In: Proceedings of the SIGCHI Conference on Human Factors in Computing Systems, CHI '04, pp. 303-310. ACM, New York, NY, USA (2004). DOI 10.1145/985692.985731. URL http://doi.acm.org/10.1145/985692.985731

37. Salvador, G., Bañó, M., Contero, M., Camba, J.: Evaluation of a distributed collaborative workspace as a creativity tool in the context of design education. In: 2014 IEEE Frontiers in Education Conference (FIE) Proceedings, pp. 1-7. IEEE (2014)

38. Salvador-Herranz, G., Camba, J.D., Naya, F., Contero, M.: On the integration of tangible elements with multitouch surfaces for the collaborative creation of concept maps. In: International Conference on Learning and Collaboration Technologies, pp. 177-186. Springer (2016) 
39. Salvador-Herranz, G., Contero, M., Camba, J.: Use of tangible marks with optical frame interactive surfaces in collaborative design scenarios based on blended spaces. In: International Conference on Cooperative Design, Visualization and Engineering, pp. 253-260. Springer (2014)

40. Schöning, J., Hook, J., Bartindale, T., Schmidt, D., Oliver, P., Echtler, F., Motamedi, N., Brandl, P., von Zadow, U.: Building interactive multi-touch surfaces. In: Tabletops-Horizontal Interactive Displays, pp. 27-49. Springer (2010)

41. Shaer, O., Hornecker, E.: Tangible user interfaces: past, present, and future directions. Foundations and Trends in Human-Computer Interaction 3(1-2), 1-137 (2010)

42. Shen, C., Everitt, K., Ryall, K.: Ubitable: Impromptu face-to-face collaboration on horizontal interactive surfaces. In: International Conference on Ubiquitous Computing, pp. 281-288. Springer (2003)

43. Suzuki, H., Kato, H.: Algoblock: a tangible programming language, a tool for collaborative learning. In: Proceedings of 4th European Logo Conference, pp. 297-303 (1993)

44. Suzuki, H., Kato, H.: Interaction-level support for collaborative learning: Algoblockan open programming language. In: The first international conference on Computer support for collaborative learning, pp. 349-355. L. Erlbaum Associates Inc. (1995)

45. Terrenghi, L., Kirk, D., Richter, H., Krämer, S., Hilliges, O., Butz, A.: Physical handles at the interactive surface: exploring tangibility and its benefits. In: Proceedings of the working conference on Advanced visual interfaces, pp. 138-145. ACM (2008)

46. Veltkamp, R.C.: Shape matching: similarity measures and algorithms. In: Shape Modeling and Applications, SMI 2001 International Conference on., pp. 188-197. IEEE (2001)

47. Weinberg, G., Gan, S.L.: The squeezables: Toward an expressive and interdependent multi-player musical instrument. Computer Music Journal 25(2), 37-45 (2001)

48. Weiser, M.: Some computer science issues in ubiquitous computing. Communications of the ACM 36(7), 75-84 (1993)

49. Wilson, F.: The Hand: How Its Use Shapes the Brain, Language, and Human Culture. Vintage Series. Vintage Books (1998). URL https://books.google.es/books?id=1_Boy_-NkwUC

50. Zuckerman, O., Arida, S., Resnick, M.: Extending tangible interfaces for education: digital montessori-inspired manipulatives. In: Proceedings of the SIGCHI conference on Human factors in computing systems, pp. 859-868. ACM (2005) 